\title{
Study on Slurry Noise of Electromagnetic Flowmeter Based on ARMA Power Spectrum Estimation
}

\author{
Song Gao and Bin Li \\ School of Mechatronics Engineering and Automation, Shanghai University, Shanghai 200072, China \\ Correspondence should be addressed to Song Gao; gaosongsan@163.com
}

Received 15 June 2015; Revised 18 October 2015; Accepted 25 October 2015

Academic Editor: Marco Mussetta

Copyright (C) 2015 S. Gao and B. Li. This is an open access article distributed under the Creative Commons Attribution License, which permits unrestricted use, distribution, and reproduction in any medium, provided the original work is properly cited.

\begin{abstract}
The output signal of the electromagnetic flowmeter measuring the slurry flow will be disturbed by the slurry noise. It is necessary to study the characteristics of slurry noise in order to reduce the interference. The methodology for the estimation of the slurry noise power spectrum based on the ARMA model is presented in this paper, and the relation among the slurry noise, the velocity, and the concentration is obtained by means of the methodology above according to the $1 / f$ characteristics of the slurry noise. The results by computer simulation experiment show that if the concentration or flow velocity is increased, then slurry noise power spectral density curve in the logarithmic coordinate system will move to the upper right; if the concentration or flow velocity is reduced, then slurry noise power spectral density curve in the logarithmic coordinate system will move to the lower left.
\end{abstract}

\section{Introduction}

The output signal of electromagnetic flowmeter for slurry flow measurement will interfere with slurry noise. The reason is at the beginning of the measuring electrode contact with the fluid and in order to resist the corrosion of electrolyte, a thin oxide film formed on the measuring electrode surface of electromagnetic flowmeter; high polarization voltage is generated between the metal and electrolyte in the process of forming oxide film, and if the material and surface state of two electrodes are the same then the polarization voltage between metal and electrolyte is changed to the commonmode disturbance voltage which has the same polarity and magnitude; when the electrode of electromagnetic flowmeter is impacted by the solid particles or fiber in the slurry, a thin oxide film on the surface of electrode is broken and generates scratches; the oxide film will regenerate; in this process polarization voltage between the electrodes and fluid will change; the common-mode disturbance voltage will become differential-mode disturbance voltage, so that the output signal of electromagnetic flowmeters is to be fluctuated; this fluctuation is a kind of interference phenomenon in the signal waveform; this phenomenon is called slurry noise. It is necessary to study the characteristics of slurry noise in order to reduce the interference.
The reasons for the slurry noise are described in [1]. This paper shows that the slurry noise is caused by the impact of the solid phase particles of the slurry on the electrode of the electromagnetic flowmeter. Reference [1] also described that slurry noise is a random signal, and the power spectral density of the signal is inversely proportional to the frequency of the signal, so they approximate the relationship to $1 / f$. Spectral analysis shows that the frequency distribution of the slurry noise is wider, and the disturbance amplitude decreased with the increase of frequency; these are the characteristics of $1 / f$. The current study [2-8] shows that the slurry noise is characterized with $1 / f$ and belongs to $1 / f$ noise, and it is shown in Figure 1.

The papers described the reasons for the occurrence of the slurry noise and the power spectrum of the slurry noise has $1 / f$ characteristics, but these papers have not studied the relationship between the flow velocity and concentration of the slurry and the slurry noise.

Zhuang and $\mathrm{Wu}[9,10]$ point out that the $1 / f$ noise is pink noise and belongs to the colored noise, and they have studied the methods of using computer simulation to simulate $1 / f$ noise. However, these methods of generating $1 / f$ noise are too complex, and the computational complexity is large. 


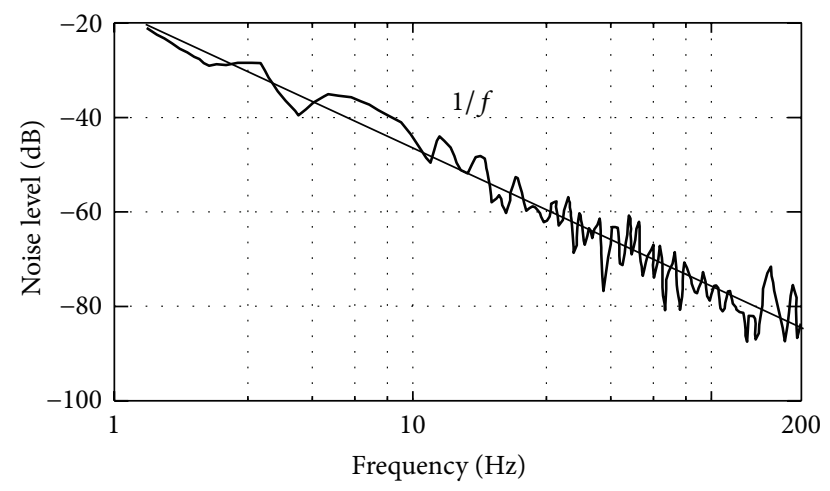

FIGURE 1: $1 / f$ characteristics of the slurry noise.

The slurry noise is a random signal, so it cannot use a clear mathematical relationship to be described; however, the power spectral density of the random signal can be estimated by the method of power spectrum estimation. In this paper, the power spectrum of the slurry noise is estimated by the method of power spectrum estimation for random signals; the relationship between the slurry noise and the velocity and concentration of the slurry is analyzed.

\section{Power Spectrum Estimation of Random Signal}

The power spectrum estimation method is the power spectral density of a random signal which is estimated with given multiple sample data, and it can be divided into classical power spectrum estimation method and modern power spectrum estimation method. The main drawback of the classical power spectrum estimation method is the fact that the frequency resolution is relatively low. The frequency resolution of modern power spectrum estimation method is relatively high, and it can be avoided by the disadvantages of classical power spectrum estimation, so it is called the high resolution spectrum estimation. The classical power spectrum estimation method assumes that the unknown data outside the data workspace is 0 , equivalent to data window. The classical power spectrum estimation method is mainly divided into correlation function method, average periodic chart method, and sliding average periodic chart method. These classical power spectral estimation methods are directly estimated by using finite length data, and there are two basic ways:

(1) Estimating the correlation function at first and then getting the power spectrum estimation by Fourier transformation.

(2) Associating with the power spectrum and square of signal amplitude frequency.

Using any of the above ways, there is a problem for the characteristic of estimation variance is not good, and the fluctuation of the estimation value along the frequency axis is very intense; the longer the data, the more serious the phenomenon.
Compared with the classical power spectrum estimation method, the modern power spectrum estimation method has the advantages of higher resolution and more accurate estimation results. So this paper chooses the modern power spectrum estimation method.

The modern power spectrum estimation method first estimates the parameters model by observing the data, and then the output power of the parameters model is calculated; finally, the signal power spectrum density is estimated. The modern power spectrum estimation method can be divided into maximum entropy spectral analysis method (AR model method), Pisarenko harmonic decomposition method, Prony extraction pole method, Prony spectral line decomposition method, and Capon maximum likelihood method.

The application of AR model is more extensive and representative. Some examples of commonly used AR models are ARMA model, AR model, MA model, and so forth. Their difference is the fact that the coefficients of the system transfer function are different. The AR model is the all poles linear model; it is also called the autoregressive model. This model is conducive to estimating the peak value of the signal power spectrum. The MA model is the all zeros linear model, and the structure of the model is simple; it is also called the sliding average model. This model is conducive to estimating the valley value of the signal power spectrum. In this paper, the study on the power spectrum of the slurry noise is not a peak value or a valley value problem of the power spectrum; the peak value and the valley value of the power spectrum must be accurately estimated. The ARMA model is the poles-zeros model; it is also called the autoregressive slip average model, and it also has the characteristics of AR and MA models. So ARMA model can accurately estimate the peak value and the valley value of the signal power spectrum, and it can fully reflect the nature of the signal power spectrum. So, the choice of the ARMA model is more suitable for the estimation of the power spectrum of the slurry noise.

Compared with ARMA, other modern power spectral estimation methods are more complex, and using these methods to estimate the power spectrum of the slurry noise, the results are not more accurate than ARMA. So, in this paper, the ARMA model is applied to estimate the slurry noise of electromagnetic flowmeter.

\section{Parameter Model for the Slurry Noise of Electromagnetic Flowmeter}

Stationary white noise signal $\varepsilon(t)$ is input to linear time invariant systems $H(t)$; the output is stationary linear colored noise $x(t)$. Its mathematical model is shown in the following equation:

$$
x(t)=-\sum_{k=1}^{K} a_{k} x(t-k)+\sum_{m=0}^{M} b_{m} \varepsilon(t-m),
$$

where $a_{k}$ and $b_{m}$ are the constant coefficients. According to (1), the noise signal $x(t)$ has a linear relationship with the stationary white noise signal $\varepsilon(t)$.

If the slurry velocity in the electromagnetic flowmeter pipeline is $V$ and the volume concentration of solid phase 
particles is $C_{s}$, then the number of solid phase particles $S$ that impact the electrode of electromagnetic flowmeter in unit time is determined. It is assumed that in a certain time $t$ the solid phase particles impacting the electrode of electromagnetic flowmeter are a uniformly distributed white noise sequence $U(n) ; S$ corresponds to the variance of white noise sequence $\sigma_{U}^{2}$; obviously, if the slurry velocity $V$ or the volume concentration of solid phase particles $C_{s}$ increases, the total number of solid phase particles impacting the electrode of electromagnetic flowmeter in unit time will also increase; namely, the variance of white noise sequence $\sigma_{U}^{2}$ increases; conversely if the slurry velocity $V$ or the volume concentration of solid phase particles $C_{s}$ decreases, the total number of solid phase particles impacting the electrode of electromagnetic flowmeter in unit time will also decrease; the variance of white noise sequence $\sigma_{U}^{2}$ decreases.

The simulation method is as follows: according to the $1 / f$ characteristics of the slurry noise, the slurry noise generated by the sequence $U(n)$ impacting the electrode is a $1 / f$ noise sequence expressed as $X(n)$. The sequence $U(n)$ can be regarded as a uniformly distributed white noise sequence, and the variance of sequence $U(n)$ is a fixed value; IIR digital filter $H(z)$ is passed by the sequence $U(n)$; a standard $1 / f$ noise sequence expressed as $X(n)$ is generated; the IIR digital filter $H(z)$ can be viewed as the process of the solid phase particles impacting the electrode of electromagnetic flowmeter. The parameters of the IIR digital filter $H(z)$ can be determined after designing it; if the concentration or flow velocity of solid phase particles is changed (i.e., the variance of sequence $U(n)$ is changed), $1 / f$ noise sequence $X(n)$ is also changed. It can be known that the characteristics of the change of slurry noise with the concentration and flow velocity by compare and analysis of the change of $1 / f$ noise sequence $X(n)$.

A model of producing the slurry noise is constructed by using the method of parameter estimation, and it is shown in Figure 2.

According to Figure 2, the number of solid phase particles that impact the electrode in a certain time is a uniform distribution sequence $U(n)$. If $U(n)$ is viewed as a stationary uniformly distributed white noise sequence, $H(z)$ is the transfer function of the system. According to (1), the parameter model of the slurry noise is obtained and expressed as follows:

$$
X(n)=-\sum_{k=1}^{p} a_{k} X(n-k)+\sum_{k=0}^{q} b_{k} U(n-k) .
$$

Set the coefficient $b_{0}=1$. If the coefficients $b_{k}(k=1,2, \ldots, q)$ equal 0 , the parameter model is the all poles model (AR model). If the coefficients $a_{k}(k=1,2, \ldots, p)$ equal 0 , the parameter model is the all zeros model (MA model). If the coefficients $b_{k}(k=1,2, \ldots, q)$ and the coefficients $a_{k}(k=$ $1,2, \ldots, p)$ are not all equal to 0 , the model is the poles-zeros model; it is also called ARMA (autoregressive slip average) model. Because the ARMA model can preferably simulate $1 / f$ characteristics of the slurry noise, the ARMA model is used to estimate the slurry noise in this paper.

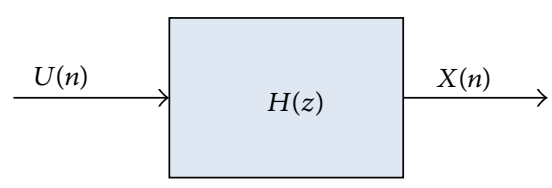

FIGURE 2: The parameter model of constructing the slurry noise.

Using Z-transformation to (2) and setting the coefficient $b_{0}=1$, then the transfer function of the system is expressed as follows:

$$
H(z)=\frac{1+b_{1} z^{-1}+b_{2} z^{-2}+\cdots+b_{q} z^{-q}}{1+a_{1} z^{-1}+a_{2} z^{-2}+\cdots+a_{q} z^{-p}}=\frac{B(z)}{A(z)} .
$$

It has both zeros and poles, and the distribution position of the poles and the zeros needs to be considered to ensure the stability of the system. Equation (3) is expressed by ARMA $(p, q) ; p$ or $q$ is the order of the model.

The power spectral density of sequence $U(n)$ is variance $\sigma_{U}{ }^{2}$; then the power spectral density of the slurry noise sequence $X(n)$ is expressed as follows:

$$
P_{X}\left(e^{j \omega}\right)=\sigma_{U}{ }^{2} \frac{B\left(e^{j \omega}\right) B\left(-e^{j \omega}\right)}{A\left(e^{j \omega}\right) A\left(-e^{j \omega}\right)}=\sigma_{U}{ }^{2}\left|\frac{B\left(e^{j \omega}\right)}{A\left(e^{j \omega}\right)}\right|^{2} .
$$

By $z=e^{j \omega}$, (5) can be obtained:

$$
P_{X}(z)=\sigma_{U}^{2} \frac{B^{2}(z)}{A^{2}(z)}=\sigma_{U}^{2} H^{2}(z) .
$$

According to (3) and (5), (6) can be obtained:

$$
P_{X}(z)=\sigma_{U}^{2}\left|\frac{1+b_{1} z^{-1}+b_{2} z^{-2}+\cdots+b_{q} z^{-q}}{1+a_{1} z^{-1}+a_{2} z^{-2}+\cdots+a_{q} z^{-p}}\right|^{2} .
$$

According to (6), the power spectral density of the slurry noise sequence within the $Z$ domain can be obtained.

According to $1 / f$ characteristics of the slurry noise, the transfer function of the slurry noise is expressed as follows [11]:

$$
H(j \omega)=\frac{1}{\sqrt{j \omega}}=\frac{1}{\sqrt{\omega}} e^{-j(\pi / 4)}
$$

or

$$
H(s)=\frac{1}{\sqrt{s}} .
$$

On the basis of (6), the power spectral density of the slurry noise is expressed as follows:

$$
P_{X}(s)=\sigma_{U}^{2}|H(s)|^{2}=\frac{\sigma_{U}^{2}}{s} .
$$

By the bilinear transformation method, the following formula can be obtained and it is shown as follows:

$$
s=\frac{2}{T} \frac{1-z^{-1}}{1+z^{-1}},
$$


where $T$ is the sampling period; depending on the bilinear transformation, the single valued mapping relationship between $S$ plane and $Z$ plane is established, and the single valued mapping relationship is unaffected by the value of $T$; the design results are not influenced by $T$ values. Thus, the value of $T$ can be set to 2 .

According to (9) and (10), (11) can be obtained:

$$
P_{X}(z)=\frac{\sigma_{U}^{2}\left(1+z^{-1}\right)}{1-z^{-1}} .
$$

According to (6) and (11), (12) can be obtained:

$$
\left|\frac{1+b_{1} z^{-1}+b_{2} z^{-2}+\cdots+b_{q} z^{-q}}{1+a_{1} z^{-1}+a_{2} z^{-2}+\cdots+a_{q} z^{-p}}\right|^{2}=\frac{1+z^{-1}}{1-z^{-1}} .
$$

The coefficients $a_{k}(k=1,2, \ldots, p)$ and $b_{k}(k=$ $1,2, \ldots, q)$ can be obtained by (12). The order of the ARMA model needs to be determined; this paper selects $p=q=5$ by several methods of order identification for ARMA models [12-15] and comparison of simulation results in this paper.

According to (12), $b_{1}=0.5, b_{2}=-0.125, b_{3}=0.063, b_{4}=$ -0.036 , and $b_{5}=0.026$ and $a_{1}=-0.5, a_{2}=-0.125, a_{3}=$ $-0.063, a_{4}=-0.036$, and $a_{5}=0.026$ can be calculated.

Therefore,

$$
H(z)=\frac{B(z)}{A(z)},
$$

where

$$
\begin{aligned}
B(z)=1 & +0.5 z^{-1}-0.125 z^{-2}+0.063 z^{-3}-0.036 z^{-4} \\
& -0.026 z^{-5} \\
A(z)= & 1-0.5 z^{-1}-0.125 z^{-2}-0.063 z^{-3}-0.036 z^{-4} \\
& +0.026 z^{-5}
\end{aligned}
$$

By (2), the ARMA model of the slurry noise sequence $X(n)$ is expressed as follows:

$$
\begin{aligned}
X(n)= & 0.5 X(n-1)+0.125 X(n-2) \\
& +0.063 X(n-3)+0.036 X(n-4) \\
& -0.026 X(n-5)+U(n)+0.5 U(n-1) \\
& -0.125 U(n-2)+0.063 U(n-3) \\
& -0.036 U(n-4)-0.026 U(n-5) .
\end{aligned}
$$

\section{Simulation of the Power Spectrum Density of the Slurry Noise}

The flow of fluid in the pipe can be divided into several layers according to the distance from the wall of pipe. The measuring electrode of the electromagnetic flowmeter is in the nearest flowing layer of the distance to the wall of pipe; the solid phase particles in this flow layer directly impact the electrode. The object of study is the impact of the solid phase particles on the electrode so we only need to consider the flow layer closest to the wall of pipe. If only two-dimensional case is considered, the flow velocity of the flow layer closest to the wall of pipe is expressed as $V^{\prime}$; the solid phase particles are composed of different shapes and sizes of particles; it can be considered that the actual diameter of the solid phase particles is Gauss distribution; the average diameter of the solid phase particles is expressed as $d_{s}$; the volume concentration of the solid phase particles closest to the wall of pipe is expressed as $C_{s}$; the diameter electrode is expressed as $d_{e}$; the number of the solid phase particles that impact the electrodes during the unit time can be calculated by the following formula:

$$
S=\frac{V^{\prime} C_{s} d_{e}}{d_{s}}
$$

Equation (16) shows that, in the unit time, the number of the solid phase particles that impact the electrode is proportional to the velocity, the concentration, and the diameter of the electrode, and it is inversely proportional to the diameter of the solid phase particle. In this paper, the diameter of the solid phase particles is fixed.

Below by simulation, the characteristics of the power spectral density of the slurry noise and its variation with the velocity and concentration of the slurry will be studied.

If the flow velocity of the flow layer closest to the wall of pipe is $V_{1}^{\prime}$, the volume concentration of the slurry is $C_{s 1}$, the impact of solid phase particles on the electrodes is a white noise sequence $U_{1}(n)$, and the number of the solid phase particles that impact the electrodes during the unit time is expressed as $S_{1}$; in this case the value of variance of the sequence $U_{1}(n)$ is $1 . U_{1}(n)$ is the excitation signal and passes $H(z)$; according to (13) and (15), the time domain waveform chart of the slurry noise sequence can be obtained, and it is shown in Figure 3.

Figure 3 shows that the fluctuations magnitude of the slurry noise in the time domain is large. In order to analyze the properties of the slurry noise, the slurry noise was influenced by the change of concentration of solid phase particles and the flow velocity; the power spectral density of the slurry is also analyzed.

According to (6), if the volume concentration of the slurry is $C_{s 1}$ and the flow velocity of the slurry is $V_{1}^{\prime}$, the power spectral density of the slurry noise in the logarithmic coordinate system is shown in Figure 4.

From Figure 4 it can be seen that the power spectral density of the slurry noise is a curve of $1 / f$ characteristic.

The influence of the concentration of the slurry on the power spectral density of the slurry noise is as follows.

If the concentration of the slurry is increased and it is expressed as $C_{s 2}$, and $C_{s 2}=2 C_{s 1}$, the flow velocity of the slurry is unchanged; according to (16), the number of solid phase particles impacting the electrode of electromagnetic flowmeter in unit time is expressed as $S_{2}$, and $S_{2}=2 S_{1}$; the solid phase particles impacting the electrode of electromagnetic flowmeter are a white noise sequence $U_{2}(n)$. Then the value of variance $\sigma_{2 U}{ }^{2}$ of sequence $U_{2}(n)$ is 2 ; the power spectral density of the slurry noise in the logarithmic 


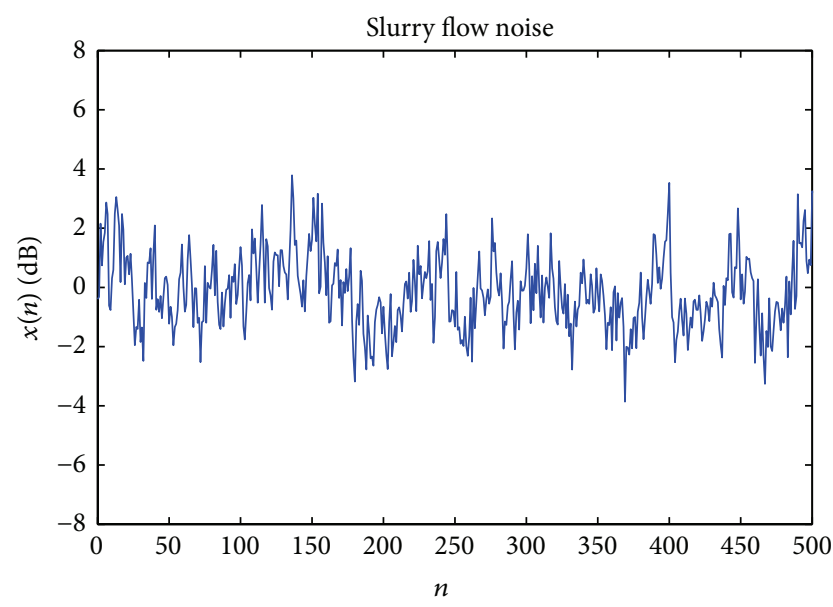

FIGURE 3: The time domain waveform chart of the slurry noise.

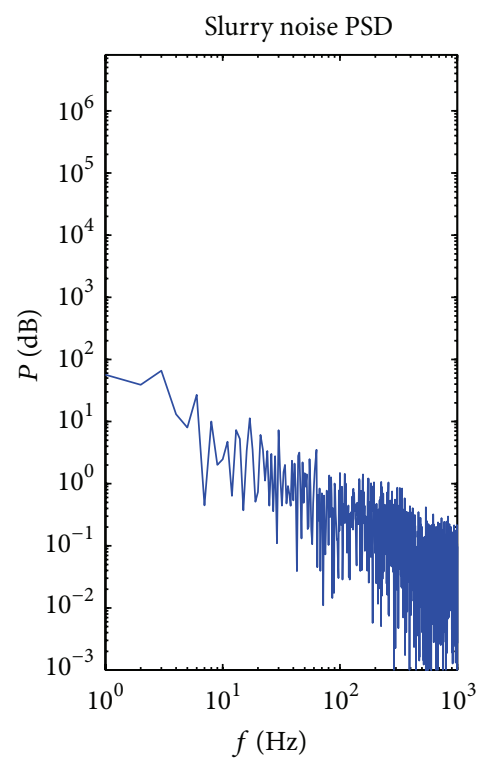

Figure 4: The power spectral density of the slurry noise.

coordinate system is shown in Figure 5(a). If the value of concentration of the slurry is kept unchanged at $C_{s 2}$, the value of flow velocity of the slurry increases to $V_{2}^{\prime}$, and $V_{2}^{\prime}=2 V_{1}^{\prime}$, so the number of solid phase particles impacting the electrode of electromagnetic flowmeter in unit time is expressed as $S_{3}$, and $S_{3}=2 S_{2}$. The solid phase particles impacting the electrode of electromagnetic flowmeter are a white noise sequence $U_{3}(n)$; the value of variance $\sigma_{3 U}{ }^{2}$ of sequence $U_{3}(n)$ is 4 ; the power spectral density of the slurry noise in the logarithmic coordinate system is shown in Figure 5(b).

According to (16), the increase of the concentration and flow velocity of the slurry caused the increase of the number of solid phase particles impacting the electrode of electromagnetic flowmeter in unit time and led to the increase of slurry noise. From Figure 4 it can be seen that the power spectral density curves of the slurry noise increase with the increase of the number of solid phase particles impacting the electrode of electromagnetic flowmeter in unit time; it shows the characteristics of the curves shifting to the upper right. The power spectral curves of the slurry noise shown in Figures 4 and 5 are shown in the same logarithmic coordinate system as that shown in Figure 6.

In Figure 6 , the bottom curve $\mathrm{A}$ represents the power spectral density of the slurry noise where the value of the slurry concentration is $C_{s 1}$ and the value of flow velocity of the slurry is $V_{1}^{\prime}$. The middle curve $\mathrm{B}$ represents the power spectral density of the slurry noise where the value of the slurry concentration increased to $2 C_{s 1}$ and the flow velocity of the slurry remains unchanged and the value of it is still $V_{1}^{\prime}$. The upper curve $\mathrm{C}$ represents the power spectral density of the slurry noise where the value of the slurry concentration is still $2 C_{s 1}$ and the value of flow velocity of the slurry increased to $2 V_{1}^{\prime}$. Figure 6 shows that the number of solid phase particles impacting the electrode of electromagnetic flowmeter in unit time increases with the increase of the slurry concentration and the flow velocity of the slurry and causes the change in power spectral density of the slurry noise; it can be seen that the power spectral density curves of the slurry noise shift to the upper right. Obviously if the slurry concentration or the flow velocity of the slurry is decreased, the power spectral density curves of the slurry noise shift to the lower left.

\section{Conclusions}

This paper is based on the background of the output signal of the electromagnetic flowmeter used in the slurry measurement which interferes with the slurry noise. For the purpose of studying the relationship between the concentration and flow velocity of the slurry and the slurry noise, the paper is based on the $1 / f$ property of the slurry noise and the method of ARMA power spectrum estimation; the ARMA model of the slurry noise is established and the slurry noise is simulated. The relationship between the slurry noise and the concentration and flow velocity of the slurry is analyzed. The following conclusions are obtained:

(1) According to the causes of slurry noise, the ARMA model can be applied to estimate the power spectrum of the slurry noise.

(2) If the flow velocity or concentration of slurry changes, the magnitude of the slurry noise will also be changed. It is reflected in the power spectral density curve of the slurry noise changes in the position of the coordinate system. If the concentration or flow velocity is increased, then slurry noise power spectral density curve in the logarithmic coordinate system will move to the upper right; if the concentration or flow velocity is reduced, then slurry noise power spectral density curve in the logarithmic coordinate system will move to the lower left.

These conclusions can provide some theoretical basis for designing the slurry type electromagnetic flowmeter. 


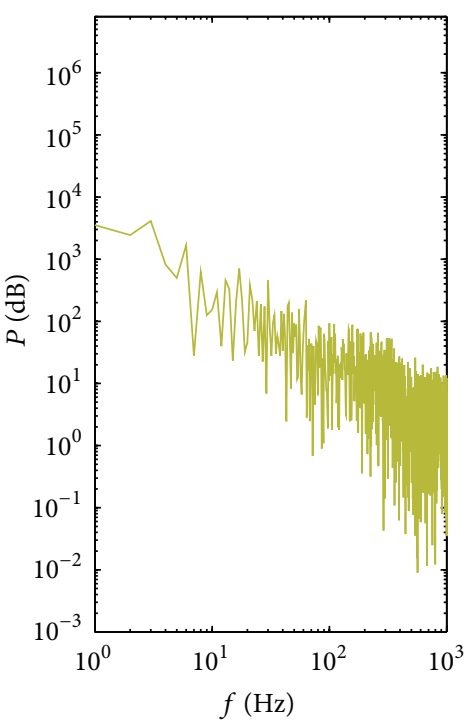

(a) Slurry noise PSD

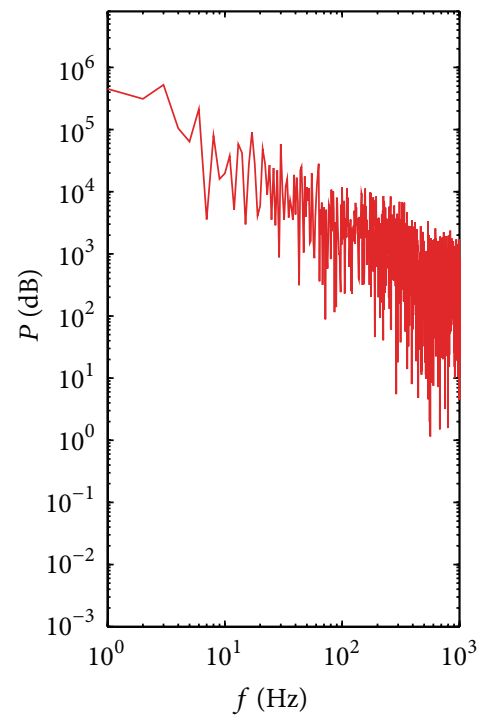

(b) Slurry noise PSD

FIGURE 5: The power spectral density of the slurry noise after the change of the flow velocity and concentration of the slurry.

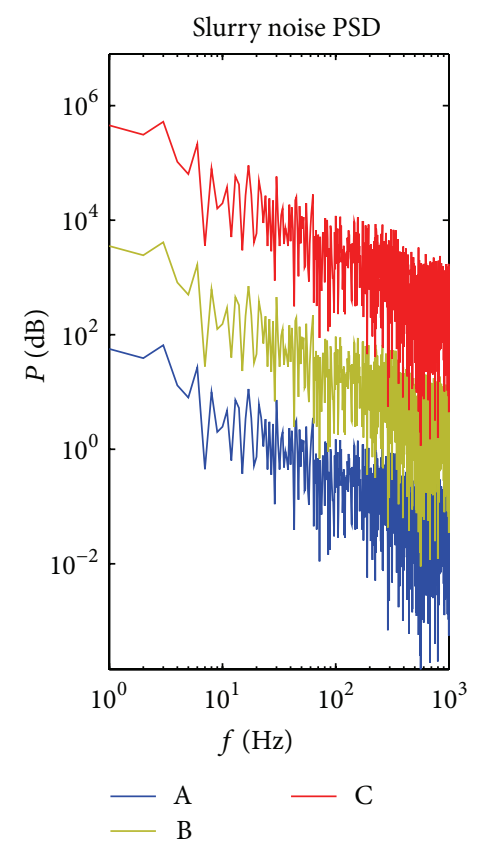

FIGURE 6: The power spectral density of the slurry noise at different flow velocity and concentration of the slurry. In curve A, concentration is $C_{s 1}$ and flow velocity is $V_{1}^{\prime}$; in curve $\mathrm{B}$, concentration is $2 C_{s 1}$ and flow velocity is $V_{1}^{\prime}$; in curve $\mathrm{C}$, concentration is $2 C_{s 1}$ and flow velocity is $2 V_{1}^{\prime}$.

\section{The Inadequacy in This Study}

For simplicity, the establishment of the ARMA model of the slurry noise in this paper only considers the influence of solid phase particles which impact one measuring electrode of electromagnetic flowmeter. In fact there are two measuring electrodes for electromagnetic flowmeter; the work of considering the case of two electrodes to establish mathematical model of the slurry noise and analyze the influence of the concentration and flow velocity of the slurry on the slurry noise needs further study.

\section{Conflict of Interests}

The authors declare that they have no conflict of interests regarding the publication of this paper.

\section{References}

[1] W. C. Cai, Z. Y. Ma, G. F. Qu, and S. L. Wang, Electromagnetic Flowmeter, China Petrochemical Press, Beijing, China, 2004 (Chinese).

[2] L. Wang, G. Wang, Z. Y. Ren, and Y. M. Tian, "Research and application of key issues of slurry type electromagnetic flowmeter in coal slurry measurement," Automation and Instrumentation, no. 6, pp. 17-24, 2013 (Chinese).

[3] L. P. Liang, Signal modeling and processing of electromagnetic flow meter for slurry flow measurement [M.S. thesis], Hefei University of Technology, Hefei, China, 2010 (Chinese).

[4] I. Wada and Yokohama, "Electromagnetic Flowmeter Utilizing Mag-netic Fields of a Plurality of Frequencies," United States, US5090250, 1990.

[5] T. Tomita, "Electromagnetic Flowmeter and Method for Electromagnetically Measuring Flow Rate," US5443552, 1994, Tokyo.

[6] S. L. Yang, K. J. Xu, L. P. Liang, R. Zhang, and G. Wang, "Development of DSP based slurry-type electromagnetic flowmeter," Chinese Journal of Scientific Instrument, vol. 32, no. 9, pp. 21012107, 2011 (Chinese).

[7] T. Tomita, "Electromagnetic Flow-Rate Measurement System," United States, US6173616B1, 1997. 
[8] Kobayashi, Kuromori, and Junto, "Electromagnetic flowmeter," China, CN 87101677A, 1989 (Chinese).

[9] Y. Q. Zhuang, Z. F. Ma, and L. Du, “The mystery over 1/f noise," China Academic Journal Electronic Publishing House, vol. 21, no. 4, pp. 69-72, 2011 (Chinese).

[10] Y. Z. Wu and M. Z. Wu, "Simulation and verification of $1 / \mathrm{f}$ noise," Ship \& Ocean Engineering, vol. 37, no. 6, pp. 38-42, 2008 (Chinese).

[11] X. B. Xu, Y. Shen, and N. Wu, "Design of IIR digital pink noise filter using genetic algorithms," Audio Engineering, vol. 56, no. 12, pp. 56-59, 2005 (Chinese).

[12] B. S. Choi, ARMA Model Identification, Springer-Verlag, New York, NY, USA, 1992.

[13] W. A. Woodward and H. L. Gray, "On the relationship between the $S$ array and the Box-Jenkins method of ARMA model identification," Journal of the American Statistical Association, vol. 76, no. 375, pp. 579-587, 1981.

[14] H. Akaike, "A new look at the statistical model identification," IEEE Transactions on Automatic Control, vol. 19, no. 6, pp. 716723, 1974.

[15] J. G. de Gooijer, B. Abraham, A. Gould, and L. Robinson, "Methods for determining the order of an autoregressive-moving average process: a survey," International Statistical Review, vol. 53, no. 3, pp. 301-329, 1985. 


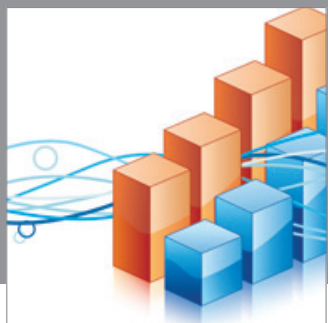

Advances in

Operations Research

mansans

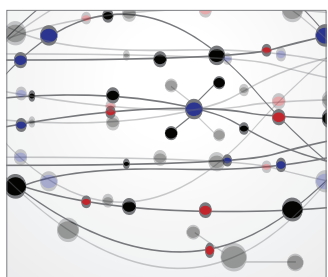

The Scientific World Journal
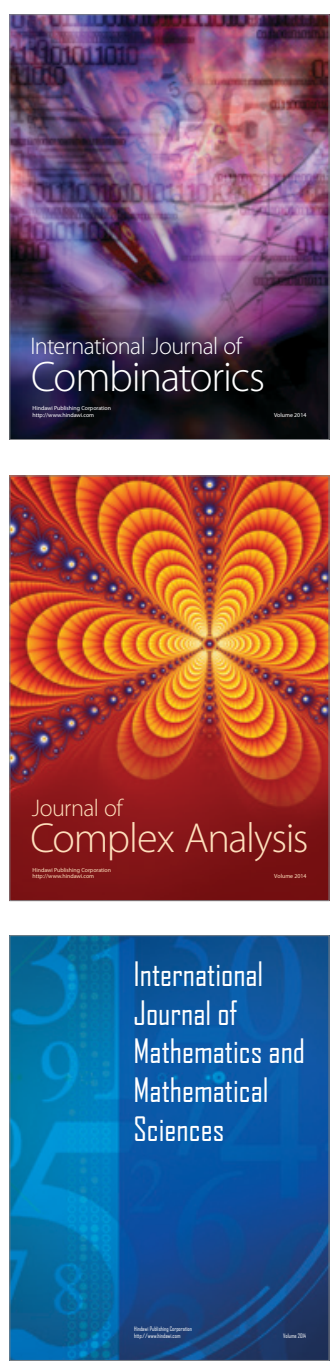
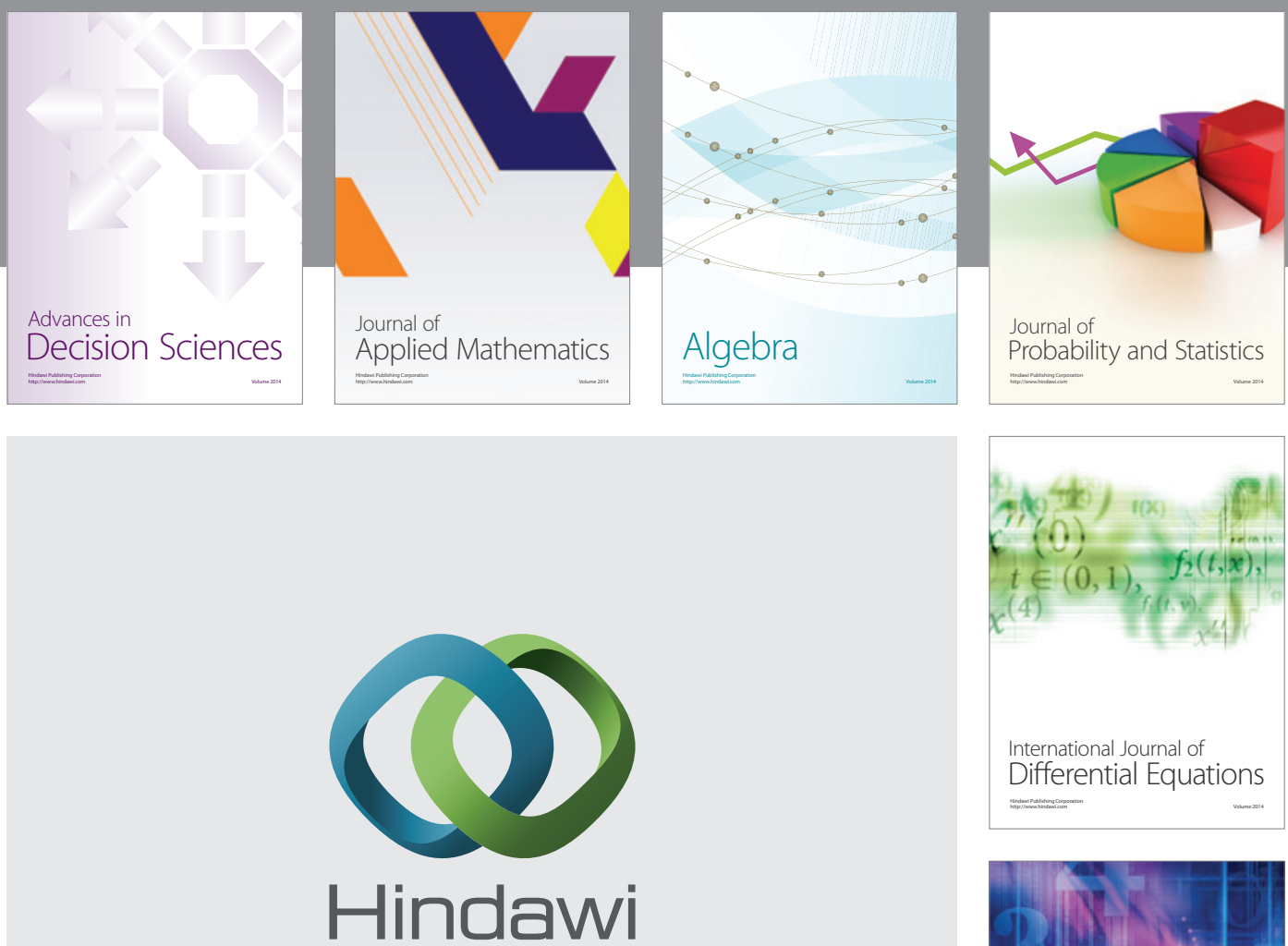

Submit your manuscripts at http://www.hindawi.com
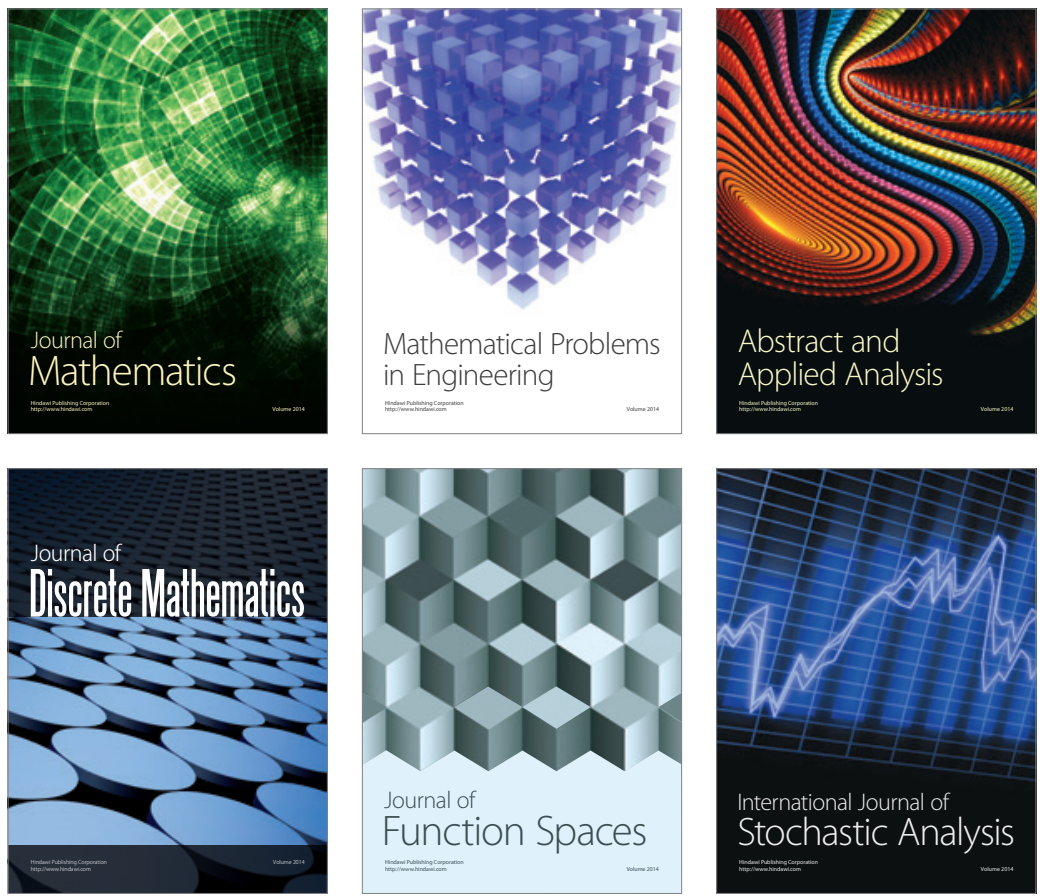

Journal of

Function Spaces

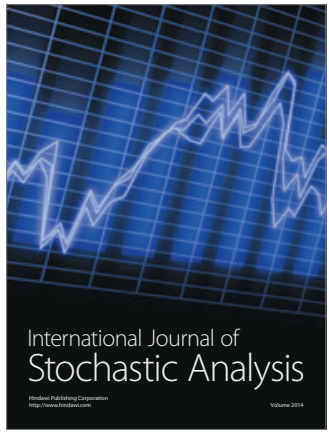

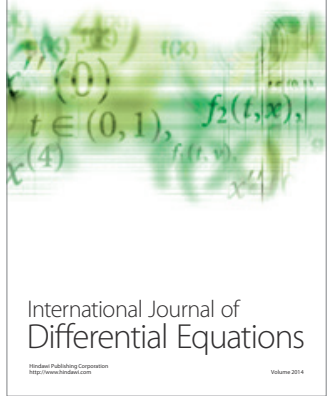
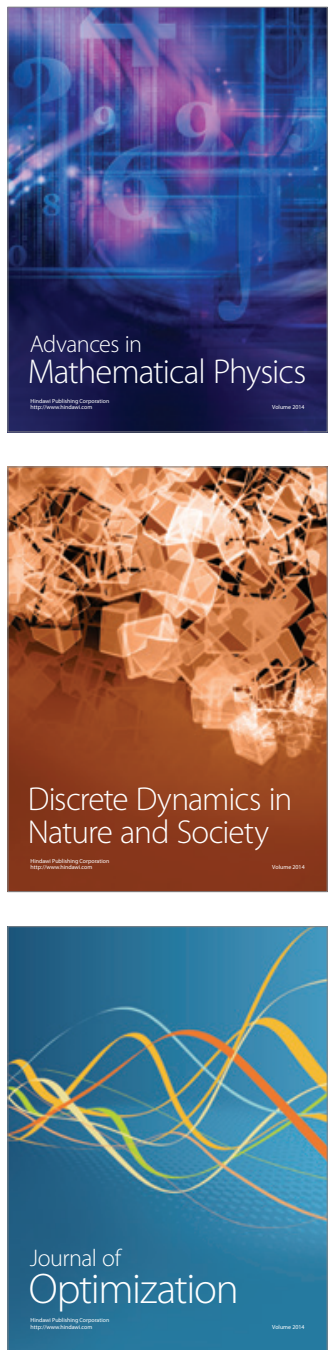